\title{
The long-term fate of epistaxis patients with exposure to antithrombotic medication
}

\author{
Stadler, Rafael R ; Kindler, Rahel ; Holzmann, David ; Soyka, Michael B
}

\begin{abstract}
The goal of this study was to evaluate independent risk factors for long-term epistaxis recurrences and their severity. Individual retrospective cohort study- $2 \mathrm{~b}$ level of evidence. The medical information of 603 emergency epistaxis patients was acquired during a former study. This cohort has been contacted 6 years later by conventional mail and asked to answer a specific paper questionnaire. The following parameters were evaluated: recurrent epistaxis episodes, need for a surgical intervention to stop the recurrent bleeding, patient's history for hypertension and diabetes, intake of hemostasis impairing medication now and in the past. One hundred and six (106) patients were included in the study (35.8 $\%$ response rate). The mean observation period was 76.58 months. Almost half of the patients (41.5\% $=44 / 106$ ) reported at least one recurrent epistaxis episode. Patients with exposure to VKA (vitamin $\mathrm{K}$ antagonists) showed significantly more frequently a recurrent epistaxis episode. The binary logistic regression confirmed the intake of VKA as an independent and significant risk factor with an odds ratio of 11.6. Every single patient who had to undergo a surgical intervention to stop a recurrent bleeding stated ASA (Acetylsalicylic Acid) intake. We provide evidence that the intake of a vitamin $\mathrm{K}$ antagonist is an independent long-term risk factor for recurrent epistaxis episodes. The intake of ASA is a risk factor for the severity of recurrent epistaxis with the increased need for a surgical intervention not only in a short- but also in a long-term perspective. Level of evidence: This prognostic investigation, designed as a combined prospective and retrospective cohort study, reaches level $2 \mathrm{~b}$ level of evidence as it includes retrospective aspects.
\end{abstract}

DOI: https://doi.org/10.1007/s00405-016-3913-9

Posted at the Zurich Open Repository and Archive, University of Zurich

ZORA URL: https://doi.org/10.5167/uzh-126073

Journal Article

Accepted Version

Originally published at:

Stadler, Rafael R; Kindler, Rahel; Holzmann, David; Soyka, Michael B (2016). The long-term fate of epistaxis patients with exposure to antithrombotic medication. European Archives of Oto-RhinoLaryngology, 273(9):2561-2567.

DOI: https://doi.org/10.1007/s00405-016-3913-9 
1 ZORA-Citation: Stadler, Rafael R; Kindler, Rahel; Holzmann, David; Soyka, Michael

2 B (2016). The long-term fate of epistaxis patients with exposure to antithrombotic

3 medication. European Archives of Oto-Rhino-Laryngology, 273(9):2561-2567.

4 ZORA-Link: http://www.zora.uzh.ch/126073/

6 Abstract:

7 Objectives/Hypothesis

8 The goal of this study was to evaluate independent risk factors for long-term epistaxis

9 recurrences and their severity.

10 Study design

11 Individual retrospective cohort study - 2b level of evidence.

12 Methods

13 The medical information of 603 emergency epistaxis patients was acquired during a

14 former study. This cohort has been contacted six years later by conventional mail

15 and asked to answer a specific paper-questionnaire. The following parameters were

16 evaluated: Recurrent epistaxis episodes, need for a surgical intervention in order to

17 stop the recurrent bleeding, patient's history for hypertension and diabetes, intake of

18 hemostasis impairing medication now and in the past.

19 Results

20 One hundred and six (106) patients were included in the study (35.8\% response

21 rate). The mean observation period was 76.58 months. Almost half of the patients

$22(41.5 \%=44 / 106)$ reported at least one recurrent epistaxis episode. Patients with

23 exposure to VKA (vitamin $\mathrm{K}$ antagonists) showed significantly more frequently a

24 recurrent epistaxis episode. The binary logistic regression confirmed the intake of

25 VKA as an independent and significant risk factor with an odds ratio of 11.6. Every

26 single patient who had to undergo a surgical intervention in order to stop a recurrent

27 bleeding stated ASA (Acetylsalicylic Acid) intake. 
29 We provide evidence that the intake of a vitamin $\mathrm{K}$ antagonist is an independent

30 long-term risk factor for recurrent epistaxis episodes.

31 The intake of ASA is a risk factor for the severity of recurrent epistaxis with the

32 increased need for a surgical intervention not only in a short- but also in a long-term

33 perspective.

34

35

36

37

38

39

40

41

42

43

44

45

46

47

48

49

50

51

52

53 
Key-Words:

58

Epistaxis, Nose bleed, Recurrence, Risk Factors, Prognosis, Prevalence, Warfarin,

60

\section{Level of Evidence:}

62 This prognostic investigation, designed as a combined prospective and retrospective 63 cohort study, reaches level $2 \mathrm{~b}$ level of evidence as it includes retrospective aspects. 64 
Introduction:

Epistaxis is a leading cause for admission to the otorhinolaryngologic emergency

room. The majority of the population will suffer a nosebleed at some point in their life.

Epidemiologic surveys showed an adult life prevalence of 60 percent for an epistaxis episode. Only every tenth case seeks medical attention. ${ }^{1}$

Besides the idiopathic episodes, where the cause remains unclear, the etiology can either be local or systemic. Concerning systemic etiologies, one must be aware of iatrogenic influences on the hematologic equilibrium through medication exposure. First and foremost important hemostasis impairing medications like vitamin $\mathrm{K}$ antagonists or antiplatelet drugs interfere with this system. Hemorrhagic side effects of oral anticoagulation or antiplatelet drugs seem intuitively apparent regarding their pharmacological characteristics and goals. ${ }^{2}$ Patients on such medication walk a tightrope between reducing the risk for thrombotic events and the inevitable risk of bleeding.

On a daily basis, we are often confronted with epistaxis patients' concerns about their course of any following bleeding episodes in the future, not only inquiring about the moment but also the following years.

100 It is important to be able to define independent risk factors and evaluate their

101 presence in order to give the patient an adequate and customized prediction. For this purpose, the exact acquirement of a patient's history is once again of utmost importance.

104 Several epistaxis risk factors have been investigated and discussed controversially

105 for many years, i.e. physical and chemical irritation, temperature and humidity, 
106 hypertension, septal pathologies and inflammatory conditions. Some proposed

107 factors were able to maintain their position and others were pushed into the

108 background. In recent years, anticoagulative and antiaggregative medication,

109 traumatic injury, alcohol consumption, hematologic disorders and long lasting

110 hypertension/arteriosclerosis could maintain their position in being relevant risks for

111 nasal bleeding. ${ }^{5,6,7,8,9}$

112 However, the impact of independent risk factors after an epistaxis episode in a long-

113 term perspective is largely unknown.

114 Based on a study of our clinic that investigated risk factors within epistaxis patients

115 presenting at the ENT (Ear, Nose and Throat) emergency ${ }^{3}$, we designed a long-term

116 follow-up survey study.

117 The goal of this study was to evaluate, whether certain independent factors could

118 help to predict any recurrences and their severity on the basis of long-term survey

119 data.

120

121

122 
124 This investigation was designed as a cohort survey study at the University Hospital of

125 Zurich. During a former study we systematically collected medical information of 603

126 emergency epistaxis patients who have been seen and treated at the unit (including

127 in hospital therapy and outpatient treatment) of our tertiary ENT department. ${ }^{3}$ The

128 treating physician obtained and stored the data electronically at a pre-programmed

129 form within the clinical information system. The data of this initial cohort was

130 prospectively obtained in the period from March 29, 2007 to April first, 2008.

131 In our clinical information system, 35 patients from the initial cohort have been

132 registered as deceased in the meantime. The remaining patients of the cohort have

133 been contacted by conventional mail in April 2014 and asked to answer a specific,

134 psychologically elaborated, but not validated, paper-questionnaire (Table I). The

135 paper-questionnaire had a multiple-choice design and could be sent back to us by

136 mail without any charges. However, participation in the survey was voluntary and

137 without any compensation.

138 The data of this survey cohort was also part of a second study estimating the long-

139 term outcome and satisfaction after the treatment at our ENT department (manuscript 140 submitted).

141 We directed our attention particularly on following parameters of the patient's medical

142 history: Any recurrent epistaxis episodes, the need for a surgical intervention in order

143 to stop the recurrent bleeding, the patient's history of hypertension and diabetes, and

144 the intake of hemostasis impairing medication now and in the past.

145 The patient was asked if he or she could recall the initial nosebleed and its treatment.

146 Additionally, he or she was asked whether the initial treatment was performed under

147 general or local anesthesia. The collected data from the paper survey was

148 transferred into a digital format, which then was imported into the statistics software. 
149 We matched these survey answers with the digitally documented protocols in our

150 clinical information system and excluded patients with incongruent data.

151 The occurrence of a recurrent epistaxis episode during the long-term observation and

152 the surgical intervention at a recurrent episode were defined as primary endpoints.

153 The statistical analyses were performed by the Statistical Package for the Social

154 Sciences software (SPSS) version 22.0.0.1 (IBM Corp., Armonk, NY, USA).

155 We performed descriptive statistical analyses and cross-tabulating Pearson Chi-

156 Square tests with asymptotic two-sided significances to evaluate differences in the

157 captured characteristics between the patients group with or without recurrent

158 epistaxis. Binary logistic regression was conducted with recurrent bleeding as

159 dependent variable and following factors as covariates: Sex, age, intake of

160 hemostasis impairing medication: ASA (Acetylsalicylic Acid), Marcoumar®

161 (Phenprocoumon), Plavix $®$ (Clopidogrel), other; patient's history of hypertension and

162 diabetes. We performed the Hosmer-Lemeshow test, which is an evaluated test to

163 determine the goodness of fit of the logistic regression model. Confidential interval for

164 the exponentiation of the B coefficient - Hazard Ratio - was defined as 95\%. Results

165 were considered statistically significant at the p-level $<0.05$.

166 The study was conducted in accordance to the latest version of the Helsinki

167 declarations and with the permission of the ethical committee of the canton of Zurich

168 (KEK-ZH-Nr.: 2013-0519, ClinicalTrials.gov-Identifier: NCT02127554). 


\section{Results:}

174 A total of 568 conventional mails have been sent to patients from the initial cohort.

175205 mails were returned by the postal service, due to impossible delivery. 28 mails

176 returned with a death notice by the relatives or carer of the patient.

177120 patients returned the filled in questionnaire by mail $(120 / 335=35.8 \%)$. These 120

178 patients answering the request originate from the initial cohort. Their data will be

179 labeled as "survey cohort". Seven patients had to be excluded from the survey cohort

180 because they did not remember the initial treatment. Four patients did remember the

181 treatment incongruently to our clinical information system. These eleven patients

182 were excluded from the study to eliminate other potentially incorrectly remembered

183 data. Additionally, one patient did not answer the survey-question about any

184 recurrent epistaxis episodes and two patients did not answer the survey-question

185 about the actual use of hemostasis impairing medication. Therefore, 106 patients

186 were analysed.

187 The survey cohort consisted of $68.9 \%$ (73) male and $31.1 \%$ (33) female patients.

188 This imbalance did not show a significant difference concerning the primary endpoint

189 of a recurrence. The mean interval between the initial cohort and the survey cohort

190 was 76.58 months (SD $4.0[71-84 \mathrm{Mt}]$ ). The initial cohort showed the following

191 distribution of hemostasis impairing medication intake: 46.2\% (49/106) none, 34.9\%

192 (37/106) ASA only, 10.4\% (11/106) VKA, 7.5\% (8/106) combined antiplatelet therapy

193 of ASA and Clopidogrel, 0.9\% (1/106) Clopidogrel only.

194 The survey cohort showed following distribution of hemostasis impairing medication

195 intake: 49.1\% (52/106) none, 36.8\% (39/106) ASA, 10.4\% (11/106) VKA, 0.9\%

$196(1 / 106)$ combined therapy of ASA and VKA, 0.9\% (1/106) antiplatelet therapy with

197 Clopidogrel, 1.9\% (2/106) other. The shift of types of treatment is depicted in Figure

198 1. Regarding the overall ASA shift in single cases reveals that 17 patients of initially 
$45(37.8 \%)$ discontinued ASA intake between the initial and the survey time point. In

200 contrast did eleven patients start to take ASA. Of those eleven patients did eight

201 individuals declare no exposure to any antithrombotic medication at the initial cohort,

202 and three individuals declared exposure to a VKA. The need for an adaptation of the

203 VKA therapy or application of Vitamin $\mathrm{K}$ in the initial cohort has been published

204 previously $^{14}$.

205 Regarding the overall VKA shift in single cases reveals that three patients of initially

206 eleven (27.3\%) discontinued VKA intake between the initial cohort and the survey

207 cohort. In contrast did four patients start to take VKA. Of those four patients did three

208 individuals declare exposure to ASA only at the initial cohort, and one individual

209 declared exposure to the combination of ASA and Clopidogrel.

$21141.5 \%$ (44/106) reported a recurrent epistaxis episode on either side of the nose.

212 Patients that declared intake of a VKA at the survey showed significantly more

213 frequently a recurrent epistaxis episode $(p=0.002) .83 .3 \%(10 / 12)$ of patients with

214 exposure to VKA suffered from a recurrent episode, while 16.7\% (2/12) did not

215 (Table 2).

216 The binary logistic regression confirmed the intake of VKA as an independent and

217 significant risk factor for a recurrent epistaxis episode with an odds ratio of 11.6

$218(p=0.007)$ (Table 3). Goodness of fit was confirmed by the Hosmer and Lemeshow

219 test $\left(p=0.882, \mathrm{Chi}^{2}=3.710\right)$.

220 No further covariate, including any other hemostasis impairing medication, showed

221 significance as an independent risk factor for a recurrent epistaxis episode.

22219 of the 44 patients $(43.2 \%)$ suffering from a recurrent bleeding declared treatment

223 of a subsequent episode by an ENT specialist. Eleven $(11 / 19=57.9 \%)$ patients have

224 sought an ENT specialist in a private practice and reported following distribution of 
225 epistaxis treatment: Eight (8) coagulations, one (1) packing, one (1) packing with

226 subsequent coagulation and one (1) did not reported the performed treatment.

227 Eight $(8 / 19=42.1 \%)$ patients have sought our tertiary ENT emergency and reported

228 following distribution of epistaxis treatment: Two (2) coagulations, Two (2) packings,

229 one (1) packing with subsequent coagulation, one (1) packing with subsequent

230 surgical intervention and two (2) surgical interventions only.

231 Every single patient (3/3) who had to undergo a surgical intervention in order to stop

232 a recurrent bleeding stated ASA intake at the initial time point. 


\section{Discussion:}

236 This cohort survey study is able to show that the intake of a VKA is a significant and

237 independent long-term risk factor for recurrent epistaxis episodes, while ASA did not

238 exert a significantly increased risk for recurrent episodes in a long-term perspective.

239 The exposure to acetylsalicylic acid did however show a link to severe recurrent

240 nosebleeds, with the need of a more invasive therapeutic revision-intervention.

241 An extrapolated calculation estimated a prevalence of $15.8 \%$ for minor recurrent

242 epistaxis episodes. ${ }^{4}$ In our cohort the recurrence rate was very high with more than

243 every third patient suffering from a recurrent episode. Several potential risk factors

244 for epistaxis have been identified and discussed controversially for many years.

245 Some proposed factors maintained their position, and others were either pushed into 246 the background or were able to gain more attention.

247 Examples for evaluated risk factors are traumatic injury, physical and chemical

248 irritation, allergic rhinitis, viral and bacterial rhinosinusitis, nasal tumors, temperature

249 and humidity or hemostasis impairment. ${ }^{5,6}$ Examples for non fully confirmed but

250 discussed potential risk factors are hypertension ${ }^{7}$, alcohol consumption ${ }^{8}$, septal spurs

251 or deviation. ${ }^{9}$

252 A study, conducted to investigate short-time recurrences showed treatment with

253 untargeted gauze packing due to an unidentified bleeding source as a significant risk

254 factor for a subsequent bleeding within a one week timeframe. ${ }^{10}$ On the contrary, did

255 other concurrently observed risk factors, i.e. patient's history for rhinitis or sinusitis,

256 septal deviation, use of antithrombotic agents or history for hypertension show no

257 significant differences. ${ }^{10}$ An investigation about the effectiveness of different

258 treatment options in epistaxis endorsed that coagulation at an anterior and surgical

259 occlusion at a posterior bleeding are able to successfully salvage failed packing 
260 therapies. ${ }^{11}$ Therefore is the appropriate choice of a treatment option of essential 261 importance in order to reduce recurrences.

262 Vitamin $\mathrm{K}$ antagonist therapy is a long-standing and widely postulated risk factor for 263 epistaxis. ${ }^{12} \mathrm{~A}$ recent, retrospective and controlled cohort study endorses the 264 suggestions and showed, that besides the exposure to Warfarin ${ }^{\circledR}$, none of the widely 265 believed risk factors for epistaxis in general, were associated with risk of recurrence 266 during a three years period. ${ }^{13}$

267 This is in accordance with our finding of Marcoumar®, a Europe-wide used VKA, as 268 an independent long-term risk factor for recurrent epistaxis episodes. Exposure to 269 VKA, especially if not regularly checked by a physician, can easily lead to INR levels 270 that are too high. In order to take appropriate action in case of a nosebleed in 271 patients who are not within optimal target value ranges, it is recommended to test the prothrombin time and INR in every epistaxis patient on VKAs. ${ }^{14}$

273 The intake of acetylsalicylic acid has recently been added to the group of risk factors.

274 Although already proposed as a risk factor in the last millennium ${ }^{15}$, it has unjustly 275 been pushed into the background by the VKA. Recently, it has been reasserted that 276 antiaggregational therapy with ASA is not only a subsidiary but a major risk factor for epistaxis and a higher severity. ${ }^{3}$ In the same study, did the exposure to ASA supersede VKA therapy as a risk factor for severe epistaxis.

279 To the best of our knowledge does the current literature not address the long-term 280 course of epistaxis patients during a timeframe of more than three years as done in 281 our investigation.

282 The exposure to VKA compared to other antithrombotic agents and potential risk 283 factors shows an odds ratio of 11.6 for recurrences. We also could support the 284 finding of exposure to ASA as a risk factor for epistaxis episodes with high severity 285 not only in a short- but also in a long-term perspective. Therefore, for patients with 
exposure to ASA an increased probability for a surgical intervention in order to stop a recurrent bleeding can be predicted. This finding is not statistically significant due to

288 its small number of cases. Nonetheless did all patients that required a surgical intervention in order to stop a recurrent bleeding declare ASA intake at the initial 290 cohort.

291 Hypertension as a potential risk factor ${ }^{7}$ is still controversially discussed and not 292 confirmed. It was repeatedly described not to increase the risk for epistaxis or its 293 severity. ${ }^{3,16}$ Acute hypertension during a nosebleed is a proposed ${ }^{17}$ and intuitively 294 apparent cause for a refractory epistaxis. Nevertheless, was acute hypertension 295 counter-intuitively not a risk factor for severe epistaxis in our own analysis of almost 296600 emergency events. ${ }^{3}$ On the long run, hypertension appears to be a weak 297 proximate risk factor, as only a (non-significant) trend was observed towards more 298 recurrences. In addition to hypertension, the covariate male sex also showed an independent statistical trend for an increased risk for epistaxis. It has been confirmed 300 that men are at risk for a more extensive coronary disease compared to women. ${ }^{18}$ 301 These findings support the idea that atherosclerosis, as a consequence of long-term 302 hypertension and thus continuous stress to the vascular system, leads to more 303 vulnerable vessels and thus bleeding and recurrences. ${ }^{19}$

304 The study is limited due to its non-controlled design and a relatively low response 305 rate of $35.8 \%(120 / 335)$. However, in such a long postinterventional timeframe and a 306 relatively old population this was anticipated and therefore a high number of more 307 than 500 patients in a mean timeframe of 76 months was chosen in order to get the 308 lowest possible bias. A further potentially limiting factor could be a selection bias. We 309 consider a selection bias as unlikely, since our cohort was large and there was no 310 obvious bias concerning the responders and non-responders. 
312 In contrast to other studies, we also acquired minor epistaxis episodes, which are

313 defined as episodes that were handled autonomously by the patient without seeking

314 help of a general practitioner or an ENT specialist. Therefore, we collected recurrent

315 episodes regardless of their severity and thus included a cohort that most likely

316 represents a very representative group of epistaxis patients. Furthermore, the

317 Hosmer and Lemeshow test indicated a good fit of our logistic regression model. 


\section{Conclusion:}

319 Recurrent bleedings after epistaxis treatment are underestimated and occur at a very

320 high rate. We provide evidence that the intake of a VKA is an independent long-term

321 risk factor for recurrent epistaxis episodes.

322 The intake of ASA is a risk factor for the severity of recurrent epistaxis episodes with

323 the increased need for a surgical intervention not only in a short- but also in a long-

324 term perspective.

325 Concerning the above mentioned increased risks, we recommend prescribing both

326 antithrombotic agents only with an indisputable indication. In line with our previous

327 studies we imperatively discourage from taking ASA as a "lifestyle drug" at any age.

328 However, further studies are needed to endorse this recommendation.

329 Patients with a high-risk profile should be informed and trained in the prevention,

330 first-aid measures and then seeking medical advice in case of recurrent epistaxis.

331 Therefore, in order to assess the patients' risk profile and to provide an accurate

332 prognosis it is once again crucial to meticulously record the patients' history.

333 Matching the patient's history with evaluated epistaxis risk factors leads to a better

334 counseling concerning recurrent episodes or their severity. 
1. Villwock JA, Jones K. Recent trends in epistaxis management in the United States: 2008-2010. JAMA Otolaryngol Head Neck Surg 2013;139:1279-84.

2. Verheugt FW, Granger CB. Oral anticoagulants for stroke prevention in atrial fibrillation: current status, special situations, and unmet needs. Lancet 2015.

3. Soyka MB, Rufibach K, Huber A, Holzmann D. Is severe epistaxis associated with acetylsalicylic acid intake? Laryngoscope 2010;120:200-7.

4. Benninger MS, Marple BF. Minor recurrent epistaxis: prevalence and a new method for management. Otolaryngol Head Neck Surg 2004;131:317-20.

5. Melia L, McGarry GW. Epistaxis: update on management. Curr Opin Otolaryngol Head Neck Surg 2011;19:30-5.

6. Kemal O, Sen E. Does the weather really affect epistaxis? B-ENT 2014;10:199-202. 7. Herkner H, Laggner AN, Müllner M, et al. Hypertension in patients presenting with epistaxis. Ann Emerg Med 2000;35:126-30.

8. Soyka MB, Schrepfer T, Holzmann D. Blood markers of alcohol use in epistaxis patients. European archives of oto-rhino-laryngology : official journal of the European Federation of Oto-Rhino-Laryngological Societies 2012;269:1917-22. 9. Melia L, McGarry G. Epistaxis in adults: a clinical review. Br J Hosp Med (Lond) 2008;69:404-7.

10. Ando Y, Iimura J, Arai S, et al. Risk factors for recurrent epistaxis: importance of initial treatment. Auris Nasus Larynx 2014;41:41-5.

11. Soyka MB, Nikolaou G, Rufibach K, Holzmann D. On the effectiveness of treatment options in epistaxis: an analysis of 678 interventions. Rhinology 2011;49:474-8.

12. Denholm SW, Maynard CA, Watson HG. Warfarin and epistaxis--a case controlled study. J Laryngol Otol 1993;107:195-6.

13. Abrich V, Brozek A, Boyle TR, Chyou PH, Yale SH. Risk factors for recurrent spontaneous epistaxis. Mayo Clin Proc 2014;89:1636-43.

14. Soyka MB, Holzmann D. Should we test the prothrombin time in anticoagulated epistaxis patients? Allergy \& rhinology 2013;4:e52-3.

15. Tay HL, Evans JM, McMahon AD, MacDonald TM. Aspirin, nonsteroidal antiinflammatory drugs, and epistaxis. A regional record linkage case control study. Ann Otol Rhinol Laryngol 1998;107:671-4.

16. Cingoz F, Oz BS, Arslan G, et al. Is chronic obstructive pulmonary disease a risk factor for epistaxis after coronary artery bypass graft surgery? Cardiovasc J Afr 2014;25:27981.

17. Terakura M, Fujisaki R, Suda T, Sagawa T, Sakamoto T. Relationship between blood pressure and persistent epistaxis at the emergency department: a retrospective study. J Am Soc Hypertens 2012;6:291-5.

18. Lansky AJ, Ng VG, Maehara A, et al. Gender and the extent of coronary atherosclerosis, plaque composition, and clinical outcomes in acute coronary syndromes. JACC Cardiovasc Imaging 2012;5:S62-72.

19. Lubianca Neto JF, Fuchs FD, Facco SR, et al. Is epistaxis evidence of end-organ damage in patients with hypertension? Laryngoscope 1999;109:1111-5. 
1. Do you remember the treatment from $\mathrm{xxx}$ ?
a. No
b. Yes

2. Was surgery in general anesthesia needed at the time?
a. No

b. Yes

3. Were any of the treatments back then painful for you?
a. No
b. Little
c. Medium
d. Strong
e. Very strong

4. Were any of the treatments back then particularly uncomfortable for you?
a. Cautery
b. Packing
c. Surgery
d. None

5. Have you experienced any complications or lasting sequelae during the treatment or later on?
a. No
b. Nasal obstruction
c. Crusting
d. Other:

6. Do you suffer from permanent sequelae after the treatment?

a. No

b. Yes:

7. Have you experienced nose bleeding after the treatment?
a. No
b. Yes, which side?
c. How many times a year?

8. Did you need medical treatment after the initial event?
a. No
b. Yes
c. When?
d. Where?

9. What was done?
a. Cautery
b. Packing
c. Surgery

10. Do you take anticoagulant medication?
a. No
b. Aspirin
c. Marcoumar ${ }^{\circledR}$ (Phenprocoumon)
d. Plavix ${ }^{\circledR}$ (Clopidogrel)
e. Other:

11. Do you have a high blood pressure?
a. No

b. Yes

12. Do you take any medication because of your blood pressure?

$$
\begin{array}{ll}
\text { a. } & \text { No } \\
\text { b. } & \text { Yes }
\end{array}
$$

13. Do you have an elevated blood glucose level (Diabetes)?

$$
\begin{array}{ll}
\text { a. } & \text { No } \\
\text { b. } & \text { Yes }
\end{array}
$$

14. Are you treated because of your blood glucose (Diabetes)?
a. No
b. Yes

15. In case of recurrent nosebleed, would you prefer nasal packing without anesthesia or surgery with general anesthesia to stop the bleeding?
a. Packing without anesthesia

b. Surgery in general anesthesia 
Table 2

Variables stratified by the incidence of a recurrent epistaxis episode.

Pearson Chi-Square tests with asymptotic two-sided significances

\begin{tabular}{|c|c|c|c|}
\hline Variables & $\begin{array}{l}\text { Recurrent } \\
\text { epistaxis } \\
(\mathrm{n}=44) \\
\end{array}$ & $\begin{array}{c}\text { No recurrent } \\
\text { epistaxis } \\
(\mathrm{n}=62)\end{array}$ & $p$ Value \\
\hline VKA (Marcoumar $\left.{ }^{\circledR}\right)$ & 10 & 2 & .002 \\
\hline ASA & 14 & 26 & .290 \\
\hline Clopidogrel & 1 & 0 & .233 \\
\hline Other antithrombotic medication & 1 & 1 & .806 \\
\hline Sex & & & .115 \\
\hline - Male & 34 & 39 & \\
\hline - $\quad$ Female & 10 & 23 & \\
\hline Mean age at the initial event (SD), years & $62.94(16.02)$ & $63.37(15.67)$ & .889 \\
\hline Patient's history for hypertension & 31 & 32 & .052 \\
\hline Patient's history for diabetes & 5 & 4 & .371 \\
\hline
\end{tabular}

Abbreviation: SD, standard deviation 
Table 3

Binary Logistic Regression with epistaxis recurrence as dependent variable.

\begin{tabular}{|c|c|c|c|c|}
\hline \multirow[b]{2}{*}{ Covariates } & \multirow[b]{2}{*}{$p$ Value } & \multirow[b]{2}{*}{ OR } & \multicolumn{2}{|c|}{$95 \% \mathrm{CI}$ for $\mathrm{OR}$} \\
\hline & & & Lower & Upper \\
\hline VKA (Marcoumar $\left.{ }^{\circledR}\right)$ & .007 & 11.587 & 1.973 & 68.057 \\
\hline ASA & .607 & .780 & .304 & 2.005 \\
\hline Clopidogrel & 1.000 & $>10$ & .000 & \\
\hline Other antithrombotic medication & .682 & 1.856 & .096 & 35.717 \\
\hline Sex & .072 & 2.549 & .919 & 7.068 \\
\hline Age at the initial event & .247 & .982 & .952 & 1.013 \\
\hline Patient's history for hypertension & .086 & 2.397 & .884 & 6.500 \\
\hline Patient's history for diabetes & .373 & 2.062 & .419 & 10.146 \\
\hline
\end{tabular}

Abbreviations: CI, confidential interval; OR, odds ratio 
452 Figure legends:

453

454 Figure 1: Shift of ASA (Acetylsalicylic Acid) and VKA (vitamin K antagonist) intake 455 between the initial cohort and the survey cohort; y-axis: 0 to 100 patients. 